\title{
Reputation and Sovereign Default
}

\author{
Manuel Amador* Christopher Phelan ${ }^{\dagger}$
}

June 21, 2017

\begin{abstract}
This paper presents a continuous time model of sovereign debt. In it, a relatively impatient sovereign government's hidden type switches back and forth between a behavioral type which cannot default and follows a set rule governing its borrowing as a function of its current debt and the price at which it can issue additional bonds, and an optimizing type which can default on the country's debt at any time. We show that in any Markov perfect equilibrium, the optimizing type mimics the behavioral type when borrowing, revealing its type only by defaulting on its debt at random times. Further, in such Markov perfect equilibria (the solution to a simple pair of ordinary differential equations) display positive gross issuances at all dates, constant net imports as long as there is a positive equilibrium probability the government is the optimizing type, and net debt repayment only by the behavioral type. For countries which have recently defaulted, the interest rate the country pays on its debt is a decreasing function of the amount of time since its last default, its total debt is an increasing function of the amount of time since its last default, and the yield curve on its debt is downward sloping. For countries which have not recently defaulted, interest rates are constant and yield curves are flat.
\end{abstract}

\section{Introduction}

This paper presents a continuous time model of sovereign debt where a sovereign government's reputation evolves over time. In the model, a relatively impatient sovereign government's hidden type exogenously switches back and forth between a behavioral type which cannot default and follows a set rule governing its borrowing as a function of its current debt and the price at which it can issue additional bonds, and an optimizing type which

${ }^{*}$ University of Minnesota, Federal Reserve Bank of Minneapolis, and NBER

${ }^{\dagger}$ University of Minnesota, Federal Reserve Bank of Minneapolis, and NBER 
can default on the country's debt at any time. We consider the government's reputation at any time to be the international lending markets' Bayesian posterior that the country's government is the behavioral (or trustworthy) type.

We show that in any Markov perfect equilibrium (the solution to a simple pair of ordinary differential equations), the optimizing type mimics the behavioral type when borrowing, revealing its type only by defaulting on its debt at random times. We first assume that the game between the country's government and outside lenders starts with no debt and with outside lenders certain the government is currently the optimizing type since this is the continuation game following a default. That is, defaulting, by definition, sets the country's debt to zero, and Bayesian updating by lenders sets the government's reputation to zero since only the optimizing type can default. (We later characterize the Markov perfect equilibrium for all starting values of the country's debt and reputation.) We show for an endogenous finite period of time $T$ after a default (or since the start of the game), an optimizing government sets a positive but finite arrival rate of defaulting which depends only on how much time has occured since the last default. Further, as this amount of time since the last default approaches $T$, the arrival rate of default approaches infinity (certain immediate default). This implies that if the amount of time since the last default is positive but less than $T$, the country has an interior reputation which increases over time but after $T$ is certainly the behavioral type.

This characterization allows us to separate between episodes where a country has recently defaulted (defined by the amount of time since the last default being less than $T$ ) and episodes where a country has not recently defaulted. For countries which have recently defaulted, we show that net imports are positive and constant over time, or that the borrower country's consumption is constant and strictly greater than the country's endowment. After $T$ units of time since the last default, the country's consumption decreases over time and net imports become negative (the country repays its debt as opposed to rolling it over), which only occurs if the country is at that point the behavioral type.

Such a Markov perfect equilibrium displays several characteristics broadly consistent with real world sovereign debt facts. First, the interest rate the country pays is a strictly decreasing function of the amount of time since the last default. This is a consequence of default being, in equilibrium, less likely the more time since the last default. This happens because although an optimizing government is more likely to default the longer it has been since the last default, whether the country's government actually is the optimizing type is less likely the longer it has been since a default and this latter effect dominates. Second, the amount of debt the country holds is a strictly increasing function of the amount of time since the last default. Finally, as in the data, the yield curve for countries which have recently 
defaulted is downward sloping since the probability of default (conditional on not having already defaulted) decreases over time, while the yield curve for countries which have not recently defaulted is flat since the probability of default is the constant probability of the behavioral type becoming the optimizing type.

\section{The environment}

Time is continuous and infinite.

There is a small open economy whose government is endowed with a unit $y$ of a consumption good.

There is countable list of potential governments of the small open economy, with alternating types. With probability $\rho_{0}$, the first government on the list is the behavioral type and with probability $\left(1-\rho_{0}\right)$, the first government on the list is the optimizing type. The list then alternates between types. At any date $t \geq 0$, only one of the potential governments is in charge. With Poisson arrival rate $\epsilon$, an optimizing type government is replaced by the next government on the list (a behavioral type). With arrival rate $\delta$, a behavioral type government is replaced by an optimizing type. Such switches are private to the government. Initially, we examine the game where $\rho_{0}=0$ (or the government is certainly the optimizing type at the beginning of the game), and later allow $\rho_{0}>0$.

The economy has, an any time $t$, an amount $b(t)$ of outstanding debt held in international financial markets. The bonds are long duration and have a coupon that decays exponentially at rate $\lambda$, an exogenous parameter controlling the maturity of the bonds. We denote by $f$ the first coupon of a newly issued bond. The coupon $t$ periods after issuance is then given by $e^{-\lambda t} f$. We will later pay particular attention to the case where $\lambda=0$, in which case bonds are consoles paying $f$ at all future dates. In this case, along with several other assumptions, we solve the model analytically in closed form. We assume international financial markets are populated by a continuum of risk neutral investors, which discount the future at rate $i>0$. Further, we normalize the coupon of the bond, $f=\lambda+i$, so that the bond price is one if default cannot occur.

We assume that the behavioral type never defaults; that is, it always sets an arrival rate equal to zero. We also assume that, as long as it is in control, the behavioral type follows a pre-specified expenditure rule. That is, the stock of debt evolves according

$$
b^{\prime}(t)=H(b(t), q(t))
$$

for some exogenous function $H$. Note that it follows from the sequential budget constraint, 
$c(t)=y-(i+\lambda) b(t)+q(t)\left(b^{\prime}(t)+\lambda b(t)\right)$, that consumption, for the behavioral type, is determined and given by

$$
c(t)=y-(i+\lambda) b(t)+q(t)(H(b(t), q(t))+\lambda b(t)) \equiv C(b(t), q(t)) .
$$

Further note that assuming consumption (or new net borrowing) is a function only of the current amount of debt and the current bond price assumes a kind of myopia on the behavioral type in that neither consumption nor borrowing depend on any calculation of future bond prices.

We impose the following further conditions on the function $H(b, q)$ (and thus, implicitly, $C(b, q))$ :

Assumption 1. $H(b, q):\left[0, \frac{y}{i+\lambda}\right] \times[0,1] \rightarrow \mathbb{R}$ is continuous, differentiable, weakly decreasing in $b$, weakly increasing in $q$, and strictly increasing in $q$ for all $(b, q) \in\left(0, \frac{y}{i+\lambda}\right) \times(0,1)$ such that $H(b, q)>0$.

Assumption 2. (bounded debt). $H(0,0) \geq 0$ and $H\left(\frac{y}{i+\lambda}, 1\right) \leq 0$.

Assumption 3. (impatience relative to outside lenders). $H\left(0, \frac{i+\lambda}{i+\lambda+\delta+\epsilon}\right)>0$.

Assumptions (1) and (2) together imply that if $b(0)=0, b(t) \leq \frac{y}{i+\lambda}$ for all $t \geq 0$. This then ensures the behavioral type's required coupon payment, $(i+\lambda) b(t)$, never exceeds $y$ and thus it is feasible for the behavioral type to never default regardless of the evolution of bond prices. A sufficient method for constructing such a function $H(b, q)$ is to let it be the solution to the deterministic maximization problem associated with a government which has any standard utility function over consumption, discounts the future at a rate strictly higher than $i+\delta+\epsilon$, has constant endowment $y$, starts with debt $b$ (which it must pay back), assumes that it will live forever and always face the bond price $q$, and must keep its debt between 0 and $\frac{y}{i+\lambda}$.

Unlike the behavioral type, an optimizing type can default. In particular, we assume an optimizing government chooses an arrival rate of default $\pi(t)$, where $\pi(t) \in \mathbb{R}_{+} \cup\{+\infty\}$. A value of $\pi(t)<\infty$ implies a zero probability of defaulting at exactly date $t$, but implies a probability of not defaulting between dates $t$ and $t+\Delta$ of $e^{-\int_{t}^{t+\Delta} \pi(s) d s}$ if $\pi(s)<\infty$ for all $s \in(t, t+\Delta)$. A value of $\pi(t)=\infty$ implies instant default at date $t$ with probability one. Thus we assume the probability of default at exactly date $t$ is either zero or one (when $\pi(t)$ is finite or infinite, respectively). In a later section, we generalize this to allow the optimizing type to choose an interior probability of defaulting at a particular date $t$. We assume that $\pi$ is right-continuous over time. 
If the government does not default, it can issue additional bonds, $b^{\prime}(t)+\lambda b(t)$, at an endogenous price $q(t)$ conditional on it having not defaulted. Again, the sequential budget constraint implies its consumption $c(t)=y-(i+\lambda) b(t)+q(t)\left(b^{\prime}(t)+\lambda b(t)\right)$.

If the government defaults, then $b(t)$ is set to zero. There are no direct costs of choosing to default and no restrictions on government borrowing from then on. In particular, it can issue additional bonds at an endogenous price $q(t)$ conditional on it having defaulted and thus $c(t)=y+q(t) b^{\prime}(t)$ (where $b(t)=0$ and $b^{\prime}(t)$ is interpreted as the right derivative of $b(t))$.

The optimizing type receives a flow payoff equal to $u(c(t))$ as long as it is continuously in power, and discounts future payoffs at rate $r>0$. We assume that $u: \mathbb{R}_{+} \rightarrow[\underline{u}, \bar{u}]$ for some finite values $\underline{u}$ and $\bar{u}$, and that $u$ is strictly increasing. We make no other assumptions on the preferences of the optimizing type. (A preview of our results is that our Markov perfect equilibrium is essentially independent of $u$ and $r$. Other than more is preferred to less, and now is preferred to later, the preferences of the optimizing type will not matter at all.)

We impose for now the following restriction on the strategies used by optimizing governments: we assume that they always choose a consumption level that is identical to that which would have been chosen by a behavioral government facing the same debt and price. The only decision left under the control of the optimizing government then is whether to default or not. We will later show that this restriction is without loss of generality: an optimizing government will have no incentive to reveal itself by choosing a consumption level different from the behavioral government, without simultaneously defaulting on its debt.

We let $\rho(t)$ represent the international market's beliefs that the government at time $t$ is the behavioral type. We assume that at time $t=0$ there is no debt, $b(0)=0$, and the government is known to be the optimizing type, that is, $\rho(0)=0$. (Again, we will later consider the case where initial debt and initial reputation are not set to zero.) Market beliefs are updated according to Bayes' rule. In case of default, $\rho(t)$ immediately jumps to zero, as the government has revealed itself to be an optimizing one, and $\rho^{\prime}(t)=\epsilon$. Conditional on no default, beliefs evolve according to standard Bayesian updating or:

$$
\rho^{\prime}(t)=(1-\rho(t)) \epsilon+\rho(t)((1-\rho(t)) \pi(t)-\delta) ; \text { if } \pi(t)<\infty,
$$

where $\rho^{\prime}(t)$ is defined to the be the right derivative of $\rho(t)$. Finally, again conditional on no default, $\rho(t)$ jumps to one if $\pi(t)=+\infty$. Note from the assumed right continuity of $\pi(t)$ that if $\pi(t)<\infty, \rho(t)$ is continuous at $t$ (since $\rho^{\prime}(t)$ is defined) and that if $\pi(t)=\infty, \rho(t)$ is right continuous at $t{ }^{1}$

\footnotetext{
${ }^{1}$ When $\rho(t)=0$ and $\pi(t)=\infty$, then Bayes' rule does not apply if no default occurs. Here, we assume $\rho$
} 
The assumptions above ensure the price of a bond is given by

$$
q(t)=\rho(t) q_{B}(t)+(1-\rho(t)) q_{R}(t)
$$

where $q_{B}(t)$ and $q_{R}(t)$ represent the expected valuation of the bond if the government is behavioral or optimizing (rational), respectively. These expected valuations solve the following pricing equations (where $q_{B}^{\prime}(t)$ and $q_{R}^{\prime}(t)$ are right derivatives):

$$
\begin{aligned}
i q_{B}(t) & =(i+\lambda)-\lambda q_{B}(t)+\delta\left(q_{R}(t)-q_{B}(t)\right)+q_{B}^{\prime}(t) \\
i q_{R}(t) & =(i+\lambda)-(\lambda+\pi(t)) q_{R}(t)+\epsilon\left(q_{B}(t)-q_{R}(t)\right)+q_{R}^{\prime}(t)
\end{aligned}
$$

with $q_{R}(t), q_{B}(t) \in[0,1]$ where $q_{R}(t)=0$ if $\pi(t)=\infty$. As before, the right continuity of $\pi(t)$ implies the continuity of $q_{B}(t)$ and $q_{R}(t)$ (and thus $q(t)$ ) at $t$ if $\pi(t)<\infty$, and the right continuity of $q_{B}(t)$ and $q_{R}(t)$ (and thus $q(t)$ ) everywhere. Since $c(t)=y-(i+\lambda) b(t)+$ $q(t)\left(b^{\prime}(t)+\lambda b(t)\right), b^{\prime}(t)=H(b(t), q(t))$, and $H$ is continuous in both arguments, that $q(t)$ is right continuous implies $c(t)$ is right continuous as well.

\section{Markov Equilibria}

We will focus attention to Markov equilibria. The payoff relevant state variables are $b(t)$ and $\rho(t)$. However, note that once an optimizing government defaults, the state $(b(t), \rho(t))$ moves back to $(0,0)$ - its original starting point. As a result, we can index Markov equilibria as functions from the time since the last default, which we denote as $\tau$.

The definition of equilibrium is as follows:

Definition 1. A Markov perfect equilibrium is a set of functions, $\{\pi(\tau), q(\tau), \rho(\tau), b(\tau)\}$, describing the optimizing government's intensity of default choice, the price of the bonds, the market belief, and the amount of bonds outstanding, all as functions of the amount of time since a previous default, such that:

1. Foreign investors break even in equilibrium:

$$
q(\tau)=\rho(\tau) q_{B}(\tau)+(1-\rho(\tau)) q_{R}(\tau)
$$

can jump to any value between zero and one inclusive. 
where $q_{B}(\tau), q_{R}(\tau) \in[0,1]$ and solve

$$
\begin{aligned}
(i+\lambda+\delta) q_{B}(\tau) & =(i+\lambda)+\delta q_{R}(\tau)+q_{B}^{\prime}(\tau) \\
(i+\lambda+\epsilon+\pi(\tau)) q_{R}(\tau) & =(i+\lambda)+\epsilon q_{B}(\tau)+q_{R}^{\prime}(\tau)
\end{aligned}
$$

2. Market beliefs are rational:

- $\rho(0)=0$ (or after a default, beliefs revert to certainty of the optimizing type) and

- conditional on no default, beliefs evolve according to Bayes' rule:

$$
\rho^{\prime}(\tau)=(1-\rho(\tau)) \epsilon+\rho(\tau)((1-\rho(\tau)) \pi(\tau)-\delta) \text { if } \pi(\tau)<\infty
$$

and $\rho$ jumps to one if $\pi(\tau)=\infty$ and $\rho(\tau)>0$.

3. The amount of debt, conditional on no default, evolves according to the behavioral's government pre-specified expenditure rule:

$$
b^{\prime}(\tau)=H(b(\tau), q(\tau))
$$

with $b(0)=0$.

4. For all times since last default $\tau \geq 0$, the default intensity of the optimizing type, $\pi(\tau)$, maximizes the optimizing type's payoff. That is, the following collection of optimal control problems (indexed by $\tau$ )

$$
V(\tau)=\sup _{\hat{\pi}: \mathbb{R}_{+} \rightarrow \mathbb{R}_{+} \cup\{+\infty\}} \int_{0}^{\infty}\left(\int_{0}^{t} e^{-(r+\epsilon) s} u(C(b(s), q(s))) d s+e^{-(r+\epsilon) t} V(0)\right) d F(t),
$$

where $F(t) \equiv 1-e^{-\int_{0}^{t} \hat{\pi}(s) d s}$, achieves a maximum with the control $\hat{\pi}(s)=\pi(\tau+s)$ for all $\tau \geq 0$.

\section{Characterizing Equilibria}

In this section, we establish the main characteristics of our Markov perfect equilibrium, again assuming $\rho(0)=0$ and $b(0)=0$. For a given $\operatorname{MPE}(\pi(\tau), q(\tau), \rho(\tau), b(\tau))$, let $V(\tau)$ and $c(\tau)$ denote the associated value to the optimizing government and consumption level as a function of time since last default, $\tau$. We first establish that for all $\tau \geq 0, V(\tau)$ is constant, and importantly, strictly greater than the value associated with the optimizing government 
consuming its endowment at all periods. We then derive the implications of this constant value on the paths of consumption $c(\tau)$, reputation $\rho(\tau)$, default intensity $\pi(\tau)$, and debt $b(\tau)$.

Lemma 1. If $(\pi(\tau), q(\tau), \rho(\tau), b(\tau))$ is a Markov perfect equilibrium with associated value $V(\tau), V(0) \geq \frac{u(y)}{r+\epsilon}$.

Proof. A possible strategy for the optimizing type is to set $\pi(0)=+\infty$. Under this strategy, his consumption always equals $C(0, q(0))$, which, since debt cannot be negative (Assumption 2), weakly exceeds $y$ for all $q(0)$.

Lemma 2. If $(\pi(\tau), q(\tau), \rho(\tau), b(\tau))$ is a Markov perfect equilibrium with associated value $V(\tau), V(\tau) \geq V(0)$ for all $\tau \geq 0$.

Proof. If $V(\tau)<V(0)$ for some $\tau \geq 0, \pi(\tau)<\infty$. A deviation setting $\pi(\tau)=\infty$ then sets the deviation value equal to $V(0)$.

Lemma 3. If $(\pi(\tau), q(\tau), \rho(\tau), b(\tau))$ is a Markov perfect equilibrium, then for all $\tau \geq 0$, there exists $t>\tau$ such that $\pi(t)>0$.

Proof. Suppose not. Then there exists $\tau \geq 0$ such that $\pi(t)=0$ for all $t \geq \tau$. This implies for all $t \geq \tau$ that $q(t)=1$. Suppose $c(t) \geq y$ for all $t \geq \tau$. If $b(\tau)>0$, then this ensures $b(t)$ eventually exceeds its upper bound, a contradiction of Assumption (2). If $b(\tau)=0, b(t)$ again eventually exceeds its upper bound since $b^{\prime}(\tau)=H(0,1)>0$ from Assumption (3). Thus there must exist $s \geq \tau$ such that $c(s)<y$. Assumptions (1) and (2) (and $q(t)=1$ for all $t \geq \tau)$ ensure that $b(t)$ is weakly increasing on $[\tau, \infty)$ and thus $c(t)$ is weakly decreasing on $[\tau, \infty)$. Thus $c(t)<y$ for all $t \geq s$, ensuring $V(s)<\frac{u(y)}{r+\epsilon}$, a contradiction of Lemma $(2)$.

Lemma 4. If $(\pi(\tau), q(\tau), \rho(\tau), b(\tau))$ is a Markov perfect equilibrium, its associated value $V(\tau)$ is continuous.

Proof. First note that $V(\tau)$ satisfies:

$$
\begin{aligned}
V(\tau) & =\sup _{T \geq 0} \int_{\tau}^{\tau+T} e^{-(\rho+\epsilon)(s-t)} u(c(s)) d s+e^{-(\rho+\epsilon) T} V(0) \\
& =\int_{\tau}^{\tau+T(\tau)} e^{-(\rho+\epsilon)(s-\tau)} u(c(s)) d s+e^{-(\rho+\epsilon) T(\tau)} V(0),
\end{aligned}
$$


for some $T(\tau) \in \mathbb{R}_{+} \cup\{+\infty\} .^{2}$ We have then that, for $\Delta>0$,

$$
\begin{aligned}
V(\tau+\Delta)-V(\tau) & \geq \int_{\tau+\Delta}^{\tau+\Delta+T(\tau)} e^{-(\rho+\epsilon)(s-(\tau+\Delta))} u(c(s)) d s+e^{-(\rho+\epsilon) T(\tau)} V(0)-V(\tau) \\
& =\int_{\tau+\Delta}^{\tau+\Delta+T(\tau)} e^{-(\rho+\epsilon)(s-(\tau+\Delta))} u(c(s)) d s-\int_{\tau}^{\tau+T(\tau)} e^{-(\rho+\epsilon)(s-\tau)} u(c(s)) d s
\end{aligned}
$$

where the inequality uses the optimality of sub-optimality $T(\tau)$ for $\tau+\Delta$. Eliminating the common terms across both integrals yields:

$$
\begin{aligned}
V(\tau+\Delta)-V(\tau) & =\{T(\tau)<\infty\} \int_{\tau+\max \{\Delta, T(\tau)\}}^{\tau+\Delta+T(\tau)} e^{-(\rho+\epsilon)(s-(\tau+\Delta))} u(c(s)) d s \\
& \geq\{T(\tau)<\infty\}\left(\int_{\tau+\max \{\Delta, T(\tau)\}}^{\tau+\Delta+T(\tau)} e^{-(\rho+\epsilon)(s-(\tau+\Delta))} d s\right) \underline{u} \\
& -\left(\int_{\tau}^{\tau+\min \{\Delta, T(\tau)\}} e^{-(\rho+\epsilon)(s-\tau)} d s\right) \bar{u}
\end{aligned}
$$

where the inequality follows from the boundedness of $u$. Solving out the integrals, we obtain

$$
\begin{aligned}
& V(\tau+\Delta)-V(\tau)=\{T(\tau)<\infty\}\left(\frac{e^{(\rho+\epsilon)(\Delta-\max \{\Delta, T(\tau)\})}-e^{-(\rho+\epsilon) T(\tau)}}{\rho+\epsilon}\right) \underline{u} \\
& -\left(\frac{1-e^{-(\rho+\epsilon) \min \{\Delta, T(\tau)\}}}{\rho+\epsilon}\right) \bar{u} \\
& \geq-\sup _{T \geq 0}\left|\frac{e^{(\rho+\epsilon)(\Delta-\max \{\Delta, T\})}-e^{-(\rho+\epsilon) T}}{\rho+\epsilon}\right||\underline{u}|-\sup _{T \geq 0}\left|\frac{1-e^{-(\rho+\epsilon) \min \{\Delta, T\}}}{\rho+\epsilon}\right||\bar{u}| \\
& =-\left|\frac{1-e^{-(\rho+\epsilon) \Delta}}{\rho+\epsilon}\right|(|\bar{u}|+|\underline{u}|)>-\infty \text {. }
\end{aligned}
$$

A similar argument provides the same lower bound for $V(\tau)-V(\tau+\Delta)$, and thus

$$
|V(\tau+\Delta)-V(\tau)| \leq\left|\frac{1-e^{-(\rho+\epsilon) \Delta}}{\rho+\epsilon}\right|(|\bar{u}|+|\underline{u}|) .
$$

Given that the right hand side is continuous and goes to zero as $\Delta \rightarrow 0$, this guarantees the continuity of $V(\tau)$.

Lemma 5. If $(\pi(\tau), q(\tau), \rho(\tau), b(\tau))$ is a Markov perfect equilibrium, its associated value, $V(\tau)=V(0)$ for all $\tau \geq 0$ such that $\pi(\tau)>0$.

\footnotetext{
${ }^{2}$ In particular, $T(\tau)=\inf \{t \geq \tau \mid \pi(t)>0\}$.
} 
Proof. Suppose $V(\tau)>V(0)$ for some $\tau>0$ such that $\pi(\tau)>0$. That $V(\tau)$ is continuous allows us to choose $\Delta>0$ sufficiently small such that $V(\tau+s)>V(0)$ for all $s \leq \Delta$. Next, note

$$
\begin{aligned}
V(\tau)= & {\left[\int_{0}^{\Delta} e^{-(r+\epsilon) s} u(c(s)) d s+e^{-(r+\epsilon) \Delta} V(\tau+\Delta)\right] e^{-\int_{0}^{\Delta} \pi(s) d s} } \\
& +\int_{0}^{\Delta}\left(\int_{0}^{t} e^{-(r+\epsilon) s} u(c(s)) d s+e^{-(r+\epsilon) t} V(0)\right) \pi(t) e^{-\int_{0}^{t} \pi(s) d s} d t
\end{aligned}
$$

where the first term on the right hand side accounts for realizations where no default occurs before time $\tau+\Delta$ and the second term accounts for realizations for which a default occurs before time $\tau+\Delta$. Next, since $\pi(\tau)>0$ (and thus from the right continuity of $\pi$ there exists $t \in(0, \Delta]$ such that $\pi(\tau+s)>0$ for all $s \leq t)$, and $V(\tau+t)>V(0)$ for all $t \leq \Delta$, if one replaces $V(0)$ with $V(\tau+t)$ in the second term (for all $t \leq \Delta$ ), this term increases. Thus a strategy of not defaulting before $\tau+t$ yields a higher value than defaulting with arrival probability $\pi(\tau+s)$ for $s \leq t$.

Lemma 6. If $(\pi(\tau), q(\tau), \rho(\tau), b(\tau))$ is a Markov perfect equilibrium with associated value $V(\tau)$. Then $V(\tau)=V(0)$, for all $\tau>0$.

Proof. Suppose there exists $\tau$ such that $V(\tau)>V(0)$. Continuity of $V$ and Lemmas (3) and (5) imply that we can find a closed interval $[t, t+\Delta]$ that contains $\tau$ such that $V(s)>V(0)$ for $s \in(t, t+\Delta)$ and $V(t)=V(\tau+\Delta)=V(0)$. Further, from Lemma (5), $\pi(s)=0$ for all $s \in(t, t+\Delta)$. If $\pi(s)=0$ for all $s \in(t, t+\Delta), q$ is strictly decreasing in $s \in(t, t+\Delta)$ (since the date at which default occurs again with positive probability is getting closer). That $q$ is decreasing over time implies that if $b^{\prime}(s)<0$ for some $s \in[t, t+\Delta]$, then $b^{\prime}(\hat{s}) \leq 0$ for all $\hat{s} \in[s, t+\Delta)$. Otherwise, that $b(s)$ is continuous ensures that if $b^{\prime}(s)$ goes from negative to positive in the interval, there must exists dates $m$ and $n$ in $(s, t+\Delta)$ such that $H(b(m), q(m))<0$ and $H(b(n), q(n))>0$ where $b(m)=b(n)$ and $q(m)>q(n)$, a contradiction.

Suppose $b^{\prime}(s)<0$ for some $s \in[t, t+\Delta)$ and thus $b^{\prime}(\hat{s}) \leq 0$ for all $\hat{s} \in(s, t+\Delta)$. Then $c(s)<y$ (and from right continuity of $c(s), c<y$ for some positive amount of time after date $s$ ) and $c(\hat{s}) \leq y$ for all $\hat{s} \in(s, t+\Delta)$. That $V(t+\Delta)=V(0) \geq \frac{u(y)}{r+\epsilon}$ then implies $V(s)<V(0)$, a contradiction. Thus $b^{\prime}(s) \geq 0$ for all $s \in(t, t+\Delta)$. 
Next suppose $b_{0} \leq b_{1}, q_{0}>q_{1}$, and $H\left(b_{0}, q_{0}\right) \geq 0$.

$$
\begin{aligned}
C\left(b_{0}, q_{0}\right)-C\left(b_{1}, q_{1}\right) & =-(i+\lambda)\left(b_{0}-b_{1}\right)+q_{0}\left(H\left(b_{0}, q_{0}\right)+\lambda b_{0}\right)-q_{1}\left(H\left(b_{1}, q_{1}\right)+\lambda b_{1}\right) \\
& \geq-(i+\lambda)\left(b_{0}-b_{1}\right)+q_{1}\left(H\left(b_{0}, q_{0}\right)+\lambda b_{0}\right)-q_{1}\left(H\left(b_{1}, q_{1}\right)+\lambda b_{1}\right) \\
& =-(i+\lambda)\left(b_{0}-b_{1}\right)+q_{1}\left[H\left(b_{0}, q_{0}\right)-H\left(b_{1}, q_{1}\right)+\lambda\left(b_{0}-b_{1}\right)\right] \\
& =-i\left(b_{0}-b_{1}\right)-\lambda\left(b_{0}-b_{1}\right)\left(1-q_{1}\right)+q_{1}\left[H\left(b_{0}, q_{0}\right)-H\left(b_{1}, q_{1}\right)\right] \\
& \geq 0
\end{aligned}
$$

where the first inequality follows from $H\left(b_{0}, q_{0}\right) \geq 0$ and $b_{0} \geq 0$; and the last inequality follows because $b_{0} \leq b_{1}$, so $-i\left(b_{0}-b_{1}\right) \geq 0,-\lambda\left(1-q_{1}\right)\left(b_{0}-b_{1}\right) \geq 0$ as $q_{1} \leq 1$, and $H\left(b_{0}, q_{0}\right) \geq H\left(b_{1}, q_{1}\right)$ by Assumption (1).

Thus $c(s)$ is weakly decreasing for $s \in[t, t+\Delta]$. Since $V(t+\Delta)=V(0)$ and $\pi(s)=0$ for $s \in(t, t+\Delta)$, this implies $V(s)$ is decreasing on $(t, t+\Delta)$. But since $V(t)=V(0)$ and $V(\tau)>V(0)$, this contradicts the continuity of $V$.

Proposition 1. If $(\pi(\tau), q(\tau), \rho(\tau), b(\tau))$ is a Markov perfect equilibrium with associated constant value $V, V>\frac{u(y)}{r+\epsilon}$.

Proof. Lemma (1) ensures $V \geq \frac{u(y)}{r+\epsilon}$. So suppose $V=\frac{u(y)}{r+\epsilon}$. This implies $c(\tau)=y$ for all $\tau \geq 0$ and thus $b(\tau)=0$ for all $\tau \geq 0$. To see this, first note that since $b(\tau)$ cannot be negative (from Assumption 2), $c(\tau)$ cannot be less than $y$ unless it is first greater than $y$. Next note if $c(0)>y$, then $V>\frac{u(y)}{r+\epsilon}$ immediately (since $\pi(0)$ can always be set to infinity). So let $\tau^{*}>0$ be such that $c\left(\tau^{*}\right)>y$ and $c(\tau) \geq y$ for all $\tau \leq \tau^{*}$. Since $c$ is right continuous, there exists $\Delta>0$ such that $c(t)>y$ for all $t \in\left[\tau^{*}, \tau^{*}+\Delta\right)$. A strategy of $\pi(t)=0$ for $t \in\left[0, \tau^{*}+\Delta\right)$ and $\pi^{*}(t)=\infty$ for $t \geq \tau^{*}+\Delta$ then delivers $V>\frac{u(y)}{r+\epsilon}$. (Thus to recap, $V=\frac{u(y)}{r+\epsilon}$ implies $c(\tau)=y$ and thus $b(\tau)=0$ for all $\tau \geq 0$.)

Next note $q_{B}(\tau) \geq \frac{i+\lambda}{i+\lambda+\delta}$ for all $\tau \geq 0$. (The right hand side is the price of a bond conditional on the behavioral type and the optimizing type defaulting immediately upon a type switch.) As a result, $q(\tau) \geq \rho(\tau) \frac{i+\lambda}{i+\lambda+\delta}$ for all $\tau \geq 0$. Thus if $\rho(\tau)=1$ for any $\tau \geq 0$, we have a contradiction of $c(\tau)=y$ for all $\tau$, since from Assumption (3), the behavioral type borrows when $b=0$ and $q \geq \frac{i+\lambda}{i+\lambda+\epsilon+\delta}$. This implies $\pi(\tau)<\infty$ for all $\tau>0$ (since $\pi(\tau)=\infty$ causes $\rho(\tau)$ to equal one immediately upon no default.)

Next, from Assumption (3), in order for $b^{\prime}(\tau)=0$ for all $\tau \geq 0$, the bond price $q(\tau)$ must always be weakly less than $q^{*} \equiv \frac{i+\lambda}{i+\lambda+\epsilon+\delta}$. Assume $q(\tau)=q^{*}$ for all $\tau \geq 0$. Since

$$
q(\tau)=\int_{0}^{\infty} e^{-(i+\lambda) t-\int_{\tau}^{t}(1-\rho(s)) \pi(s) d s}(i+\lambda) d t
$$


$q(\tau)$ can be constant o

nly if the probability of default, $x(\tau) \equiv(1-\rho(\tau)) \pi(\tau)$, is constant in which case

$$
q(\tau)=\frac{i+\lambda}{i+\lambda+x}
$$

Thus if $q(\tau)=q^{*}=\frac{i+\lambda}{i+\lambda+\epsilon+\delta}$ for all $\tau \geq 0$, then $x(\tau)=\epsilon+\delta$ for all $\tau \geq 0$. Further, since $\rho^{\prime}(\tau)=(1-\rho(\tau)) \epsilon+\rho(\tau)(x-\delta)$ is linearly increasing in $x$, and the bond price, $q$, is monotonically decreasing in $x$, the maximum bond price consistent with no borrowing, $q^{*}$, corresponds to the minimal amount of learning conditional on no default. Substituting $x=\epsilon+\delta$ into $\rho^{\prime}(\tau)=(1-\rho(\tau)) \epsilon+\rho(\tau)(x-\delta)$ delivers $\rho^{\prime}(\tau)=\epsilon$. That is, if the probability of default is set sufficiently high to cause no borrowing on the part of the behavioral type, the posterior $\rho(\tau)$ becomes an increasing linear function of time, implying that eventually $\rho(\tau)=1$, a contradiction. (If $\rho(\tau)=1, x(\tau)=(1-\rho(\tau)) \pi(\tau)$ cannot equal $\epsilon+\delta$ as assumed for finite $\pi(\tau)$.)

The only remaining possibility is that $q(\tau) \leq q^{*}$ for all $\tau$, but $q(\tau) \neq q^{*}$ for some $\tau \geq 0$, and thus $x(\tau)$ is not constant. Proving that this too causes $\rho(\tau)$ to eventually equal one is surprisingly involved, and we leave this to the appendix.

Lemma 7. $\pi(0)<\infty$.

Proof. Suppose $\pi(0)=\infty$. Then $q(0)=0$, which implies $c(0)=y$ and $V^{*}=\frac{u(y)}{r+\epsilon}$ which contradicts Proposition (1).

Lemma 8. $c(\tau) \leq c^{*} \equiv u^{-1}\left((r+\epsilon) V^{*}\right)$, for all $\tau \geq 0$.

Proof. Suppose there exists $\tau$ such that $c(\tau)>c^{*}$. Right continuity of $c$ implies there exists $\Delta>0$ such that $c(t)>c^{*}$ for all $t \in[\tau, \Delta)$. Next, set a deviation $\pi$ such that $\pi(t)=0$ for all $t \in[\tau, \Delta)$ and $\pi(\tau+\Delta)=\infty$. This deviation strategy delivers payoff greater than $V^{*}$.

Lemma 9. $c(\tau)=c^{*} \equiv u^{-1}\left((r+\epsilon) V^{*}\right)$, for all $\tau \geq 0$ such that $\pi(\tau)<\infty$.

Proof. Suppose there exists $\tau$ such that $c(\tau)<c^{*}$ and $\pi(\tau)<\infty$. Right continuity of $\pi$ and $c$ implies there exists $\Delta>0$ such that $c(t)<c^{*}$ for all $t \in[\tau, \Delta)$. This contradicts $V(\tau)=V^{*}=\frac{u\left(c^{*}\right)}{r+\epsilon}$ for all $\tau$ and $c(\tau) \leq c^{*}$ for all $\tau$.

Lemma 10. Let $T=\sup \left\{t \mid c(s)=c^{*}\right.$ for all $\left.s \leq t\right\}$. Then $0<T<\infty$ and $\pi(T)=\infty$.

Proof. That $\pi(0)<\infty$ and right continuity of $\pi$ ensures $T>0$. That $c^{*}>y$ and that debt remains bounded (Assumption (2)) implies eventually $c(\tau)<c^{*}$ and thus $\pi(\tau)=\infty$. Right continuity of $\pi$ then implies $\pi(T)=\infty$. 
Lemma 11. $\lim _{\tau \rightarrow T^{-}} \rho(\tau)=1$.

Proof. First note $q(\tau)=\rho(\tau) q_{B}(\tau)+(1-\rho(\tau)) q_{R}(\tau)$ and that $q_{B}(\tau)$ and $q_{R}(\tau)$ both are continuous at $\tau=T$ (with $q_{R}(T)=0$ and $\lim _{\tau \rightarrow T^{-}} q_{R}(T)=0$ from $\pi(T)=\infty$.) Thus given $\rho(T)=1$, if $\lim _{\tau \rightarrow T^{-}} \rho(\tau)<1$, this implies $q(\tau)$ jumps up at $\tau=T$. Debt conditional on no default, $b(\tau)$, on the other hand, is continuous at $\tau=T$. Since $H(b, q)$ is assumed strictly increasing in $q$ for all $(b, q)$ such that $H(b, q)>0$ (which it is since $\left.c^{*}>y\right), b^{\prime}(\tau)=$ $H(b(\tau), q(\tau))$ must jump up at $\tau=T$, which implies $c(\tau)$ jumps up at $\tau=T$. Since $c(\tau)=c^{*}$ for all $\tau$, this contradicts Lemma (8).

Lemma 12. For all $\tau \geq T, \pi(\tau)=\infty$.

Proof. Suppose there exists $\tau>T$ such that $\pi(\tau)<\infty$ and let $\tau^{*}=\min \{\tau \mid \tau>T$ and $\pi(\tau)<$ $\infty\}$. That $\pi$ is right continuous ensures this minimum exists and there exists $\Delta>0$ such that $\pi(t)<\infty$ for all $t \in\left[\tau^{*}, \tau^{*}+\Delta\right)$. Lemma (9) then implies $c(t)=c^{*}$ for all $t \in\left[\tau^{*}, \tau^{*}+\Delta\right)$. Further, since $\rho\left(\tau^{*}\right)=1$ (from $\pi\left(\tau^{*}-s\right)=\infty$ for a neighborhood $s>0$ ), $\rho^{\prime}\left(\tau^{*}\right)=-\delta$. Right continuity of $\pi$ then ensures there is a neighborhood to the right of $\tau^{*}$ such that $\rho(\tau)$ is decreasing. Thus in this neighborhood debt, $b(\tau)$ is increasing (since consumption is constant and greater than $y$ ). This implies that in this neighborhood, the bond price $q(\tau)$ must be increasing (or else consumption cannot be constant from Assumption (1)).

Note $q^{\prime}(\tau)=\rho(\tau) q_{B}^{\prime}(\tau)+\rho^{\prime}(\tau) q_{B}(\tau)+(1-\rho(\tau)) q_{R}^{\prime}(\tau)-\rho^{\prime}(\tau) q_{R}(\tau)$. Next, since $\rho\left(\tau^{*}\right)=1$ and $\rho^{\prime}(\tau)=(1-\rho(\tau)) \epsilon+\rho(\tau)((1-\rho(\tau)) \pi(\tau)-\delta), \rho^{\prime}(\tau)=-\delta$. Thus, $q^{\prime}\left(\tau^{*}\right)=q_{B}^{\prime}\left(\tau^{*}\right)-$ $\delta\left(q_{B}(0)-q_{R}(0)\right)$. From $(1), q_{B}^{\prime}\left(\tau^{*}\right)=(i+\lambda)\left(q_{B}\left(\tau^{*}\right)-1\right)+\delta\left(q_{B}(0)-q_{R}(0)\right)$ and thus $q^{\prime}\left(\tau^{*}\right)=(i+\lambda)\left(q_{B}\left(\tau^{*}\right)-1\right)<0$, a contradiction.

Lemma 13. $\pi(0)>0$.

Proof. At $\tau=0$, consumption is constant at $c^{*}>y$ and debt is increasing (again since $\left.c^{*}>y\right)$. Suppose $\pi(0)=0$. Note $q^{\prime}(\tau)=\rho(\tau) q_{B}^{\prime}(\tau)+\rho^{\prime}(\tau) q_{B}(\tau)+(1-\rho(\tau)) q_{R}^{\prime}(\tau)-\rho^{\prime}(\tau) q_{R}(\tau)$. Next, since $\rho(0)=0$ and $\rho^{\prime}(\tau)=(1-\rho(\tau)) \epsilon+\rho(\tau)((1-\rho(\tau)) \pi(\tau)-\delta), \rho^{\prime}(0)=\epsilon$. Thus, $q^{\prime}(0)=$ $q_{R}^{\prime}(0)+\epsilon\left(q_{B}(0)-q_{R}(0)\right)$. From $(2), q_{R}^{\prime}(0)=(i+\lambda+\pi(0)) q_{R}(0)-(i+\lambda)-\epsilon\left(q_{B}(0)-q_{R}(0)\right)$. Substituting for $q_{R}^{\prime}(0)$ and imposing $\pi(0)=0$ implies $q^{\prime}(0)=(i+\lambda)\left(q_{R}(0)-1\right)$ since $q_{R}(0)<1$ (from $T$ finite). Consumption constant when debt is increasing and the bond price is decreasing contradicts Assumption (1).

Together, these results give a tight characterization of all Markov Perfect equilibria. For $\tau \leq T$, consumption is constant and strictly larger than $y$, and from there decreases. Further, the arrival rate of default $(1-\rho(\tau)) \pi(\tau)$ is smoothly increasing from an amount strictly greater than zero to $\delta$, the arrival rate of default after date $T$. Thus the bond price 
$q(\tau)$ smoothly increases over time since the last default as well. Since consumption is greater than the endowment while it is constant, debt is smoothly increasing as well. At date $T$, the bond price $q$ becomes constant, but since $q(\tau)$ is continuous, net borrowing $b^{\prime}(\tau)$ remains positive for some time as well. Further, since $b^{\prime}(\tau)$ is continous, debt cannot go from growing to shrinking without first hitting $b^{\prime}(\tau)=0$. Thus, debt $b(\tau)$ must be weakly increasing.

To actually solve for an equilibrium, one simply solves the following set of two ordinary differential equations in $\tau$ for a guessed starting value of $q(0)$ (or $c(0))$ :

$$
b^{\prime}(\tau)=H(b(\tau), q(\tau))
$$

and, from the fact that consumption is constant between $\tau=0$ and $\tau=T$, and thus $0=C_{b}(b(\tau), q(\tau)) b^{\prime}(\tau)+C_{q}(b(\tau), q(\tau)) q^{\prime}(\tau)$, we have

$$
q^{\prime}(\tau)=\frac{C_{b}(b(\tau), q(\tau))}{C_{q}(b(\tau), q(\tau))} H(b(\tau), q(\tau))
$$

Given $b(0)=0$ and a guessed $q(0)$, these equations map out a path for $(b(\tau), q(\tau))$. Next, from $q^{\prime}(\tau)=(i+\lambda+(1-\rho(\tau)) \pi(\tau)) q(\tau)-(i+\lambda)$, we know that

$$
(1-\rho(\tau)) \pi(\tau)=\frac{1}{q(\tau)}\left((i+\lambda)(1-q(\tau))-\frac{C_{b}(b(\tau), q(\tau))}{C_{q}(b(\tau), q(\tau))} H(b(\tau), q(\tau))\right),
$$

which implies

$$
\rho^{\prime}(\tau)=(1-\rho(\tau)) \epsilon+\rho(\tau)\left(\frac{1}{q(\tau)}\left((i+\lambda)(1-q(\tau))-\frac{C_{b}(b(\tau), q(\tau))}{C_{q}(b(\tau), q(\tau))} H(b(\tau), q(\tau))\right)-\delta\right) .
$$

Thus from $\rho(0)=0$, the path of $(b(\tau), q(\tau))$ implies a path for $\rho(\tau)$. The equilibrium construction problem then is to find $(q(0), T)$ such that $\rho(T)=1$ and $q(T)=\frac{i+\lambda}{i+\lambda+\delta}$ (where the latter is the long run bond price and thus $q(\tau)$ is continuous as is necessary.) For there to be multiple Markov equilibria, there would have to multiple $(q(0), T)$ where these ending conditions are satisfied. We have not seen this in practice when computing examples. Further, in practice, if $q(0)$ is chosen too low, then at the point $T$ such that $\rho(T)=1$, $q(T)<\frac{i+\lambda}{i+\lambda+\delta}$, and if $q(0)$ is chosen too high, $q(T)>\frac{i+\lambda}{i+\lambda+\delta}$, with exactly one $q(0)$ such that when $\rho(T)=1, q(T)=\frac{i+\lambda}{i+\lambda+\delta}$.

Note that nowhere in these differential equations are any parameters associated with the preferences of the optimizing type. The preferences of the optimizing type - its rate of time preference, and its utility function (and thus risk aversion) — are not relevant. The reason is that, in equilibrium, it faces no consumption variation either across states of nature (the 
realizations of arrivals of its Poisson default events ) or across time.

\subsection{Early game play:}

To this point, we have assumed our game starts with $\rho(0)=0$ and $b(0)=0$. This is the relevant subgame after the first and any subsequent defaults. We now turn to characterizing Markov perfect equilibria for starting values of $(b, \rho)$ other than $(0,0)$, and use these results to establish that mimicing the borrowing behavior of the behavioral type is indeed optimal for the optimizing type (or that the optimizing type chooses to reveal its type only by defaulting).

First, since $b(\tau)$ in the $(0,0)$ subgame is increasing over time, for all $\left(b_{0}, \rho_{0}\right)$ such that $b_{0} \leq \sup _{\tau} b(\tau)$, there exists $\tau^{*}$ such that $b_{0}=b\left(\tau^{*}\right)$. Next, suppose $0<\rho_{0}<\rho\left(\tau^{*}\right)$, or that the optimizing type starts out with a reputation strictly lower than it will have, for debt level $b_{0}$, anytime in the subgame following a default. Here, we propose the optimizing type immediately defaults with probability $\gamma$ such that

$$
\rho\left(\tau^{*}\right)=\frac{\rho_{0}}{\rho_{0}+\left(1-\rho_{0}\right)(1-\gamma)}
$$

Since $0<\rho_{0}<\rho(\tau)$, then $\gamma \in(0,1]$, and this default behavior ensures that $(b, \rho)$ jumps either to $(0,0)$ (in the case of immediate default) or $\left(b\left(\tau^{*}\right), \rho\left(\tau^{*}\right)\right.$ ) (in the case of no immediate default). The optimizing type then follows the default strategy from either $\tau=0$ or $\tau=\tau^{*}$. He is willing to set $\gamma$ between zero and one because his continuation value is the same in either case.

If $\rho_{0}=0$, we propose the optimizing type immediately defaults probability one. Here, Bayes' rule doesn't apply for calculating beliefs conditional on not defaulting thus we are free to set the belief. Setting $\rho$ conditional on no default to $\rho\left(\tau^{*}\right)$ again ensures the optimizing type's payoff is the same regardless of whether he defaults or not and is thus willing to set $\gamma$ to one. Note that the $\rho_{0}=0$ case confirms mimicking as a best response as well: That is, if an optimizing type chooses to set net borrowing, for a given bond price, not equal to $H(b, q)$, it reveals itself to be the optimizing type, which implies immediate default.

Finally, assume $\rho_{0}>\rho\left(\tau^{*}\right)$, or that the optimizing type starts out with a reputation strictly higher than it will have, for debt level $b_{0}$, anytime in the subgame following a default. Here, we propose the optimizing type sets the default intensity $\pi$ to zero for $\hat{\tau}$ periods. His reputation $\rho$ at date $t$ is then

$$
\hat{\rho}(t)=\frac{\epsilon}{\epsilon+\delta}+e^{-(\epsilon+\delta) t}\left(\rho_{0}-\frac{\epsilon}{\epsilon+\delta}\right),
$$


which converges continuously to $\frac{\epsilon}{\epsilon+\delta}$. Since in the $(0,0)$ subgame, $\rho(\tau)$ moves continuously from zero to one, this ensures there exists date $\hat{\tau}$ such that $\hat{\rho}(\hat{\tau})=\rho(\hat{\tau})$. From date $\hat{\tau}$ on then, the optimizing type follows the $(0,0)$ equilibrium. Since from time zero to time $\hat{\tau}$, the bond price $q$ is strictly less than the bond price for that level of debt in the $(0,0)$ equilibrium, consumption along this path is strictly greater, ensuring the optimizing type is willing to set $\pi=0$ before date $\hat{\tau}$.

\section{An Example}

This section presents a computed example to illustrate the nature of Markov equilibria in our model. The parameters of our model are the endowment level $y$, the switching probabilities $\epsilon$ and $\delta$, the outside world discount rate $i$, the coupon debt maturity parameter $\lambda$, and the net borrowing function of the behavioral type $H(b, q)$ (which together imply the consumption function of the behavioral type $C(b, q)=y-(i+\lambda) b+q(H(b, q)+\lambda b)$.)

Here, we normalize $y=1$, and choose our other parameters relative to a unit of time being one year. Thus if we set $\epsilon=.01$ and $\delta=.03$, this implies a $1 \%$ chance that an optimizing government dies in the next year to be replaced by a behavioral government, and a $3 \%$ chance a behavioral government dies to be replaced by an optimizing government. (And thus the country has a behavioral government $25 \%$ of the time.) We set the outside world discount rate $i=.004$ and $\lambda=.2$, corresponding to a yearly principle payoff of $20 \%$ or roughly 5 year debt. These imply that in the long run (after date $T$ is reached and thus the government is certainly the behavioral type), the probability of default is $3 \%$ per year (from $\delta=.03$ ) and thus the long run interest rate is $3.4 \%$ (from $i+\delta=.034$ ) and the long run bond price is $.872=\frac{i+\lambda}{i+\lambda+\delta}$ (as opposed to a bond price of one if lending were riskless). For the behavioral type's borrowing function $H(b, q)$ and its corresponding consumption function $C(b, q)$, we chose

$$
H(b, q)=\left(.15-\frac{i+\lambda(1-q)}{q}\right)\left(\frac{y}{i+\lambda}-b\right) .
$$

This is exactly what falls out of a deterministic optimization problem of a country with $\log$ utility and a discount rate of .15 who believes it can sell debt at the constant bond price $q$ with the exception that we set net borrowing proportional to $\left(\frac{y}{i+\lambda}-b\right)$ while in that deterministic problem, net borrowing is proportional to $\left(\frac{y}{i}-b\right)$.

Figure 1 displays some relevant time paths for these parameters (again, where all paths start over given a default). Here, it takes about 31 years for the market belief that the government is a behavioral type to go from $\rho=0$ to $\rho=1$. In this time, debt goes from $b=0$ to about 3.5 times the country's endowment to, eventually, five times its endowment, 
Bond price, $q$

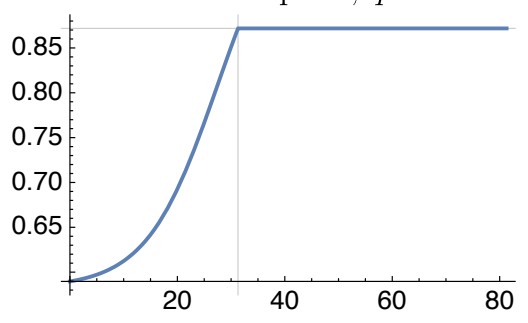

Consumption, $c$

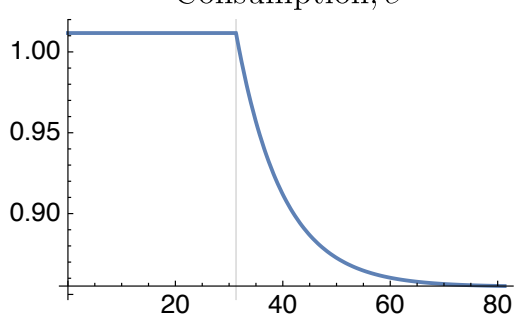

Default intensity,$\pi$

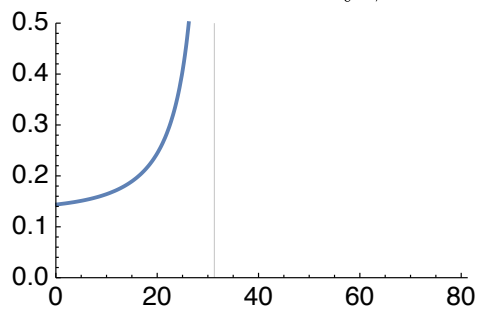

Debt, $b$

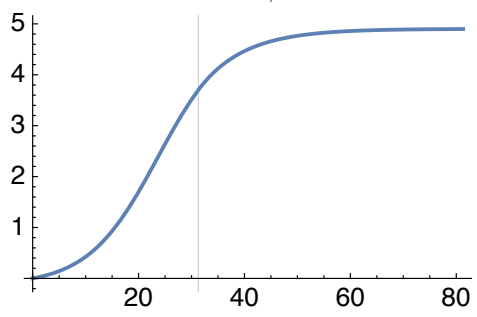

bond yield, $\frac{i+\lambda}{q}-\lambda$

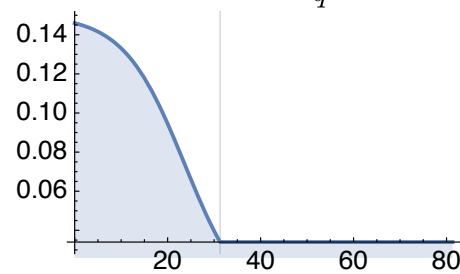

Trade deficit, $100(c-y) / y$

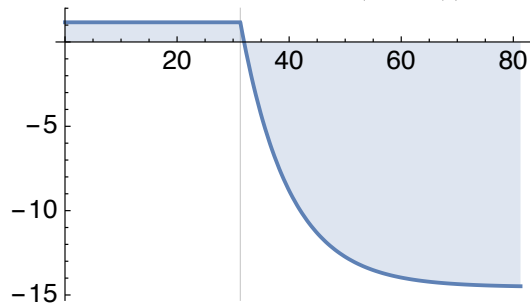

Market Belief, $\rho$

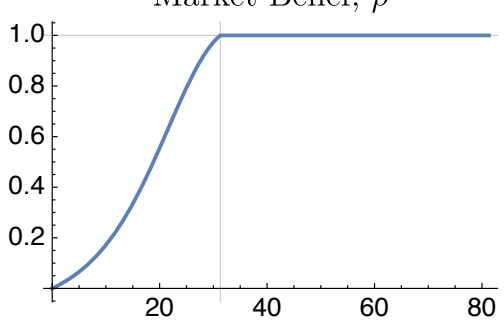

10 year - 2 year yield

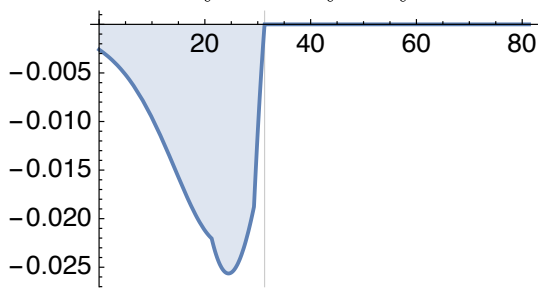

Default prob $(1-\rho) \pi$

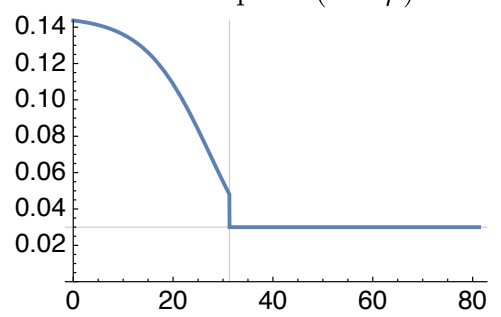

Figure 1:

while the bond price goes from .6 to its long run value of .87. Consumption stays steady at about $1 \%$ above endowment for these 31 years, and then smoothly decreases over the next 30 years to about $85 \%$ of the country's endowment. The country's default rate starts at about $14 \%$ per year, decreasing to, eventually, $3 \%$ per year. Finally, the country's yield curve (which we calculate as the 10 year zero coupon bond yield less the 2 year zero coupon bond yield) is negative, or downward sloping, for the first 31 years after a default, and zero (or flat) after that. 